\title{
An Introduction to the BIO_SOS Project: BIOdiversity Multi-SOurce Monitoring System - from Space to Species
}

\author{
Palma BLONDA, on behalf of the BIO_SOS consortium \\ CNR-ISSIA, Bari/Italy·blonda@ba.issia.cnr.it
}

This contribution was double-blind reviewed as extended abstract.

\begin{abstract}
The BIO_SOS project (www.biosos.eu) focuses on developing a robust system for habitat mapping and monitoring, named EODHaM, with this based on prior generation of land cover/use (LC/LU) and change map maps and their subsequent translation to categories of habitat appropriate to support conservation agencies and land managers in decisions relating to protection of Natura 2000 sites. The input data sources are multi-seasonal EO measurements and on-site data, including ancillary information and in-field measurements. For this purpose, the Food and Agricultural Organisation (FAO) Land Cover Classification Scheme (LCCS) and the General Habitat Categories (GHCs), from which Annex I Habitats can be defined, have been proposed for describing LC/LU and habitat categories.
\end{abstract}

\section{Introduction}

The main objective of BIO_SOS is the development of a knowledge-based pre-operational ecological modelling system suitable for effective and timely multi-annual monitoring of Natura 2000 sites and their surrounding areas particularly exposed to different and combined type of pressures. Its input data sources are satellite-based measurements and on-site data. Ontologies and semantic network are used to formally represent the expert knowledge. The proposed system, named EO Data for Habitat Monitoring (EODHaM), is compliant with on-going GEOSS, GMES and INSPIRE initiatives. Study areas in three Mediterranean and two Western Europe Countries are under way. To extrapolate from European test cases such that the methods can be applied more generally, additional areas are being considered in two tropical countries (i.e., Brazil and India), where the Natura 2000 system does not exist, but the availability of advanced monitoring systems is particularly important for Biodiversity conservation. BIO_SOS has made progress steps towards developing:

- Novel pre-operational automatic high spatial resolution (HR), very high spatial resolution (VHR) and hyper-spectral resolution EO data understanding techniques for land cover/use (LC/LU) map and LC/LU change map generation as an improvement of GMES core services.

- New GMES down-stream services based on ecological modelling, at habitat and landscape level, to combine EO and in-situ data and provide HR and VHR General Habitat 
Categories (GHCs) (http://www.ebone.wur.nl) and Annex 1 Habitat maps, their changes and adequate indicators of biodiversity.

- Quantitative landscape pattern analysis (QLPA) framework to produce composite, site/scale specific indicator set for monitoring and Natura 2000 buffer area identification.

- Ecological niche modelling (ENM) to evaluate the importance of GHCs as environmental variables to explain the distribution of the target species better than LC/LU.

- Threat analysis framework to use EO data to extract pressure trends through direct detection or monitoring of impacts on landscapes, land cover/habitat types, communities and species.

- BIO_SOS metadata geoportal.

\section{Methods for LC/LU and Habitat Mapping}

The EODHaM proposed system is a 3-stage processing system and will be described during the presentation. It has adopted the Food and Agriculture (FAO) Land Cover Classification System (LCCS) scheme and taxonomy for LC/LU class identification because LCCS is more suitable than CORINE as the land cover/use categories can be more readily translated to habitat categories (TOMASELLI et al. 2013). EODHaM $1^{\text {st }}$ stage provides robust classification of bi-temporal radiometrically calibrated EO images into LCCS Levels 1 to 2, with this based primarily on spectral data, followed by a $2^{\text {nd }}$ stage that additionally utilises contextual information to discriminate and map classes in LCCS level 3 and beyond. Based on expert knowledge of botanists, ecologists and local site managers, land cover/use and habitat classes are described by the experts in terms of their temporal characteristics and/or spatial relationships which are used in both $2^{\text {nd }}$ and $3^{\text {rd }}$ third EODHaM stages. The outputs of the $2^{\text {nd }}$ stage are LC/LU maps. The outputs of the $3^{\text {rd }}$ stage include General Habitat Categories (GHCs), Annex 1 habitats as well as biodiversity indices and their trends. Thematic change maps are also provided. Once LC/LU classes and habitats are described through a semantic language, any site can theoretically be mapped and subsequently monitored over time.

The expert knowledge classification approach adopted in BIO_SOS strongly involves end users. Consequently, the products proposed will be more familiar to the end users as they are built on their expertise and can be improved as they further engage with the process.

\section{Results}

The main project results achieved so far are synthetized in this section with reference to the project Work Packages (WP) and Deliverables (D), with this published on the BIO_SOS web page (www.biosos.eu).

WP2. For each of the sites, pre-existing information and datasets were obtained and site management authorities have been contacted and involved in the development of the EODHaM system. The structure and composition of the landscape at each site and also the human pressures and threats have been identified by stakeholders and experts (D2.2). A 
workshop was held to define stakeholder needs and to select appropriate biodiversity indicators (D2.1).

WP3. The EODHaM system architecture was defined (D3.1) and the workflow execution environment prototyped (D3.2, D3.3).

WP4. Metadata on pre-existing datasets were collected and an evaluation of their quality and relevance to the project was undertaken (D4.1). A metadata geoportal was also developed and a methodology for evaluating the quality of new spatial datasets was implemented (D4.5). Two new modules have been developed within the WebGIS platform for metadata management and sharing, to enhance communication.

Criteria were defined for the selection of EO data considered most appropriate for mapping and monitoring habitats (D4.4). Connections to previous and on-going projects were also established (D4.2).

Protocols for in-field collection of data were defined (D4.3). Training sessions on in-field protocols relevant to the identification of GHCs were held in Italy, Wales, Greece and Portugal involving also local users. In-field campaigns were performed in several sites.

WP5. The main achievement has been the implementation of a standardised procedure for classifying Land Cover/Use (LC/LU) classes according to the Food and Agriculture Organisation (FAO) Land Cover Classification System (FAO-LCCS) from very high resolution (VHR) satellite and airborne (i.e., LiDAR) and translating these to general habitat categories (GHCs), from which Annex I classes can be extracted. EODHAM system was prototyped using commercial object-orientated software but then transferred for use within open source software (i.e. Python). The system can now be applied more rapidly and consistently across sites although future refinement of the programs is needed to ensure robustness and transferability between sites. Based on the use of spectral indices, the EODHaM $1^{\text {st }}$ stage can produce classification of vegetation/non-vegetation or aquatic/terrestrial surfaces (i.e., FAO-LCCS level 1 to level 2 classes) (D5.3). Based on contextsensitive features, the EODHaM $2^{\text {nd }}$ stage modules which had been applied to several sites can classify FAO-LCCS Levels 3 and beyond. The approach is being developed with reference to ontologies for LC/LU class description. Tests have also been carried out to generate translation ontologies of LCCS into GHC classes within Protégé. The DempsterShafer theory of evidence has been collated to handle uncertainty in the data and rules (D5.1, D5.2, D5.5).

Hyperspectral data have been analysed in Italy and Wales. Whilst hyperspectral data may be beneficial for classification of particular species types/groups, the amount of information available from time-series of VHR data allows equivalent or better classifications to be generated (D5.4).

WP6. A comparison of Habitat classification systems and LC/LU taxonomies (CORINE and IGBP, FAO-LCCS), without precedent for Mediterranean, was carried out. FAO-LCCS was considered the most appropriate for subsequent LC/LU into habitat maps translation (D6.1). Disambiguation rules were implemented for LC/LU to GHC habitats translations for the implementation of the EODHaM $3^{\text {rd }}$ stage (D6.10).

Quantitative landscape pattern analysis framework was developed to produce composite, site/scale specific indicator set for monitoring and buffer area identification (D6.3). Proto- 
cols were defined for comparative habitat and landscape modelling across sites to relate biodiversity to habitat quality, linking in-situ and EO data (D6.2, D6.4). Based on Ecological Niche modelling (ENM), GHCs as environmental variables appeared better than $\mathrm{LC} / \mathrm{LU}$ to explain the distribution of the target species (D6.6, D6.7).

Threat analysis framework was established to use EO data to extract pressure trends and impacts (D6.8).

A new runoff connectivity model was proposed with R-script to calculate runoff connectivity. A new spatially implicit model for studying the effects of migration corridor size reduction on cyclic population and provide predictive biological information at a reduced computational cost with respect to explicit models was designed.

WP7. Archive and new EO data were pre-processed for several sites and fed as input to WP5.

WP8. The web page of the project has been set and continuously updated. Quality Assessment Plan (QAP) is established and implemented. An exploitation team was appointed. The first policy brief was produced. Several papers to conferences and journals have been submitted, some already published.

\section{Conclusions}

The products and systems generated by the BIO_SOS project will be made available for policy decision making and planning (e.g., scenario building) and, more specifically, evaluating the consequences of changes within Natura 2000 sites and their surroundings. EODHaM is compliant with on-going GEOSS, GMES and INSPIRE initiatives and its outputs will be used for: a) following up impact of existing and new policy. b) Defining a buffer area for each Natura 2000 site and preventing habitat fragmentation in its surrounding area. c) The use of BIO_SOS results is not limited to the management of Natura 2000 sites but to the integration of the sustainable management of the regional Natura 2000 network within the ordinary planning system. d) Innovative planning activity at a local level must try to modifying a static municipal planning system into a dynamic planning system. That means that indicators are needed to establish whether the goals associated with the approved municipal plan were met during the implementation phase. Such indicators should provide a dynamic monitoring of the different planning processes and an evaluation of the effectiveness of the policies implemented. e) Outside Europe as well, in mega-diverse countries such as Brazil and India, the operational flexibility that is required given the diversity of environments, habitats, species and threats is supported by the BIO_SOS project in that the approach is multi-resolution and multi-scale for mapping out the land cover. EODHaM system would enable managers to understand the pressure issues in multiple ecological and human environments. 